Revue d'histoire de l'enfance « irrégulière »

Le Temps de l'histoire

15 | 2013

Enfances déplacées. (II) en temps de guerre

\title{
(Lecture de) Des enfants venus de loin. Histoire de l'adoption internationale en France
}

Dominique Dessertine

\section{(2) OpenEdition \\ Journals}

Édition électronique

URL : http://journals.openedition.org/rhei/3542

DOI : 10.4000/rhei.3542

ISSN : $1777-540 \mathrm{X}$

Éditeur

Presses universitaires de Rennes

Édition imprimée

Date de publication : 30 octobre 2013

Pagination : 208-211

ISBN : 978-2-7535-2896-3

ISSN : $1287-2431$

Référence électronique

Dominique Dessertine, « (Lecture de) Des enfants venus de loin. Histoire de l'adoption internationale en France », Revue d'histoire de l'enfance « irrégulière » [En ligne], 15 | 2013, mis en ligne le 30 octobre 2013, consulté le 22 septembre 2020. URL : http://journals.openedition.org/rhei/3542 ; DOI : https:// doi.org/10.4000/rhei.3542

Ce document a été généré automatiquement le 22 septembre 2020.

(c) PUR 


\title{
(Lecture de) Des enfants venus de loin. Histoire de l'adoption internationale en France
}

\author{
Dominique Dessertine
}

\section{RÉFÉRENCE}

Paris, Armand Colin, 407p. ISBN 9782200249748

1 Depuis les années 1950, 500000 enfants ont été adoptés dans le monde hors du pays où ils sont nés. Si l'on rapporte le nombre d'arrivées d'enfants à sa population, la France est le premier des pays d'accueil et avec 100000 enfants étrangers adoptés depuis une cinquantaine d'années, elle n'est devancée que par les États-Unis. Ces données démographiques suffiraient à elles seules à justifier l'entreprise d'Yves Denéchère, qui apporte avec cet ouvrage la première synthèse sur un phénomène social complexe, trop souvent abordé à la seule aune de l'émotion. Professeur d'histoire contemporaine à l'université d'Angers, Yves Denéchère est aussi un des auteurs de notre revue. Dans ce livre, il mobilise archives publiques, nationales (ministère des Affaires étrangères), locales (conseil général du Maine-et-Loire), rapports parlementaires, littérature juridique, presse et témoignages publiés. À cette documentation quasiment exhaustive, il a ajouté les témoignages d'une trentaine de personnes, s'exprimant au nom d'associations ou en leur nom propre de parents adoptants ou d'adoptés. Sa synthèse met en valeur tous les enjeux qui se croisent autour de l'adoption internationale, histoire familiale personnelle, protection de l'enfance, destins individuels, géopolitique mondiale.

2 Le livre est structuré en trois parties, cernant les étapes essentielles de l'adoption internationale pratiquée en France. «Le temps des pionniers » remonte aux origines de l'entre-deux-guerres et aux premiers cadrages juridiques des années 1950. L'auteur rappelle que l'adoption, prévue par le Code civil en 1804, ne pouvait concerner que des 
adultes. L'adoption d'enfants (loi de 1926) est une des solutions proposées au drame des orphelins de la première guerre mondiale. Mais peu de familles se sentent concernées et seul un millier d'enfants (français) retrouvent, par ce biais, chaque année, un foyer (français). La seconde guerre mondiale est l'autre étape marquante de ce processus. Des milliers d'enfants nés de mères allemandes et de soldats français se retrouvent abandonnés. La France les rapatrie à la fin des hostilités et parfois les fait adopter. Certes, il ne s'agit pas d'adoption internationale stricto sensu mais d'un déplacement d'enfants d'un pays à l'autre. "La Famille adoptive Française", issue des œuvres sociales des cheminots, naît dans ce contexte-là. Le phénomène s'intensifiant au lendemain de la guerre, les organisations internationales interviennent. En 1952, l'OMS tente d'inscrire l'adoption internationale dans la protection de l'enfance. Le Conseil de l'Europe fait rédiger une convention sur ce thème en 1967 qui fait la part belle aux législations des pays d'accueil. Le cadrage qui s'amorce accompagne encore mal des pratiques individuelles empiriques, relayées par des congrégations religieuses ou des filières protestantes (Terre des Hommes, orphelinat coréen...).

3 Le second temps est celui des années 1970 où l'adoption internationale devient un "phénomène de société ", titre de la seconde partie du livre. Les pays sources sont de plus en plus nombreux. À la Corée, l'Inde, s'ajoute la Colombie, etc. La France réagit en mettant en place un Conseil supérieur de l'adoption, grâce entre autres à l'intervention de Simone Veil. Une première conférence mondiale sur l'adoption se tient à Milan en 1971, à laquelle participent 41 pays. En 1976 l'avis favorable de la DDASS devient obligatoire pour les familles désirant accueillir un enfant étranger. Les familles adoptantes et leurs associations sont de plus en plus parties prenantes des débats. Le Conseil supérieur estime toutefois que s'il n'est pas question d'interdire, il ne faut pas non plus encourager ce type d'adoption. Mais de nouveaux pays deviennent à leur tour pays sources. Le Brésil succède à la Corée du sud en 1987 comme premier pays de naissance. "L'adoption internationale est en question " (titre du chapitre 7) montre quelques scandales qui alimentent le débat (Salvador).

Dans la troisième partie apparaît "Le temps de la régulation" des années 1990 et suivantes. La réflexion amorcée à la fin des années 1980 sur les Droits de l'enfant démontre que si les grands principes de l'adoption internationale ont été précisés dans les années 1945-1967, leur application reste délicate. La MAI (Mission de l'adoption internationale) par exemple manque de moyens. Quatre personnes seulement suivent chaque année au moins un millier de dossiers. La Convention internationale des droits de l'enfant de 1989 affirme que toute mesure protectrice doit prendre en compte l'intérêt «supérieur » de l'enfant, et conclut que tout enfant adopté à l'étranger doit bénéficier des mêmes garanties qu'en cas d'adoption nationale. Il y a urgence, d'autant que la chute du Mur fait accéder les pays de l'Est (Roumanie, Ukraine, Hongrie, etc...) à la liste des pays proposant des enfants, que le nombre de familles candidates se multiplient et que la presse et la télévision n'hésitent pas à dénoncer, avec plus ou moins de preuves, les pratiques douteuses d'achats d'enfants ou de trafics d'organes. La Convention de La Haye en 1993 traduit la volonté de construire un cadre de normes matérielles et de coopération internationale pour mieux gérer l'adoption internationale, "promouvoir les bonnes pratiques et éradiquer les mauvaises ». Elle exige l'intervention d'une autorité centrale dans tous les pays concernés, pays de naissance, comme pays d'accueil. Pour la ratifier (1998), la France adapte sa législation en suivant les apports et les recommandations du rapport Mattei (1995). Désormais l'agrément demandé aux familles sera établi selon des normes nationales et non plus 
laissé aux appréciations divergentes des conseils généraux. Les « œuvres » deviennent des « organismes » d'adoption. Bien que n'effectuant jamais plus du tiers des adoptions en France, elles sont de plus en plus contrôlées et invitées à se regrouper. Mais la situation n'est pas clarifiée pour autant. L'adoption «bâton de pèlerin » se poursuit et en outre beaucoup de pays ne signent pas la Convention de La Haye. C'est le cas du Vietnam, pourtant premier pays pourvoyeur d'enfants, dans des conditions telles que la France suspend les adoptions dans ce pays en 1999, elles ne reprendront que l'année suivante, après la signature d'une convention bilatérale.

5 Tout au long de l'ouvrage, l'auteur accorde toute leur place aux hommes et aux femmes impliqués dans cette aventure humaine. L'adoption internationale est née d'histoires individuelles, de liens personnels, d'interconnaissances et de pratiques très empiriques. La France de l'Ouest imprégnée de catholicisme y a joué un rôle notable. Yves Denéchère n'oublie pas pour autant la grande question qui traverse constamment cette pratique. Faut-il trouver une famille pour un enfant ou un enfant à des parents? Il souligne les préoccupations des associations humanitaires et des responsables politiques qui se refusent à céder aux impulsions charitables du grand public face aux catastrophes naturelles (tremblement de terre d'Haïti) ou politiques (éclatement de la Yougoslavie). Il rappelle constamment le rôle des médias sur ce thème surtout depuis une trentaine d'années (adoptions par des " people », scandales en Roumanie, etc..).

6 L'auteur relève en conclusion que si le nombre d'adoptions internationales diminue depuis quelques années (près de 4200 en 2005, quelque 3500 en 2010), les problèmes restent les mêmes: place de l'adoption dans les politiques de protection, assainissement des réseaux pourvoyeurs, interrogation sur les relations Nord-Sud. Plus neufs sont les problèmes posés par les homosexuels et ceux de la recherche des origines. L'ouverture de nouvelles archives plus récentes et ajoutons-nous l'accès à des témoignages d'adoptés, amenés à se multiplier du fait de l'arrivée à l'âge adulte des cohortes denses des années 1990, pourront encore affiner le dossier.

7 Un livre foisonnant donc, mêlant constamment aventures personnelles et grande histoire géopolitique et soulignant combien il reste délicat d'établir des règles claires et mondiales dans cette histoire spécifique de la filiation. 